\title{
A New Noninvasive Blood Pressure Recorder Employing a Liquid-Filled Cuff
}

\author{
Osamu Tochikubo, Eiji Miyajima, Kohsuke Minamisawa, \\ Yoshiyuki Kawano, and Masao Ishii*
}

\begin{abstract}
The systolic and diastolic blood pressures (BP) measured by the conventional auscultatory method tend to be lower and higher respectively than the corresponding values obtained with invasive direct measurement. This study aims to develop a new, noninvasive device employing a liquid-filled cuff to mea sure BP accurately. The new BP recorder employs hydraulic pressure instead of air pressure in a double cuff (outer cuff and inner cuff with an implanted pressure transducer) surrounding the upper arm. The basic principle of this method is based on evidence that the pulsatile pattern of the pressure tracings obtained changes when the inner-cuff pressure reaches diastolic BP and changes again when it exceeds systolic BP. BP values measured directly from the brachial artery were compared with those obtained simultaneously by the conventional auscultatory method or by our new method in 7 normotensives and 14 hypertensive patients. The differnce in systolic BP between the auscultatory and direct methods was $-6.8 \pm 4.0$ (SD) $\mathrm{mmHg}$ and the diastolic BP difference was $1.1 \pm 6.5 \mathrm{mmHg}$. In contrast, the systolic BP difference between the new method and the direct method was $-0.6 \pm 2.0 \mathrm{mmHg}$ and that for diastolic BP was $0.6 \pm 1.9 \mathrm{mmHg}$. These values were significantly smaller than the differences obtained with the auscultatory method $(p<0.05)$. Improved conduction of brachial artery pulsation through the liquid-filled cuff to the transducer apparently improved the analysis of arterial pulsation. This new device employing a liquid-filled cuff could be useful for assessing hypertension. (Hypertens Res 1992; 15: 163-169)
\end{abstract}

Key Words: direct blood pressure, auscultatory method, accuracy of indirect blood pressure, blood pressure measurement

Blood pressure (BP) measurement is one of the most important examinations in the diagnosis and treatment of cardiovascular diseases, especially hypertension. Fundamentally, the common auscultatory method for measuring BP utilizes Magendie's reservoir manometer developed in 1850 , the brachial cuff invented in 1896 by Riva-Rocci, and Korotkoff sounds discovered in 1905 by Korotkoff (1). In spite of minor alterations, for almost a century, no major basic advances have been made in the auscultatory method aside from automation. Although comparatively accurate measurements can be obtained quickly by this simple and inexpensive method, many investigators have found that auscultatory assessment generally underestimates the direct systolic BP (SBP) and overestimates the direct diastolic BP (DBP) (2-6).

Several investigators have compared direct and indirect DBP measured at Korotkoff muffling point (D4) and cessation of Korotkoff sounds (D5). Ragen and Bordley (7) and Roberts et al (8) noted that the difference between indirect D4 values and direct DBP values was smaller than for D5 values.
Some investigators have reported a closer correlation between indirect D5 and direct DBP values, and current recommendations indicate D5 as the best estimate of indirect DBP (9). However, the mean measurement error (auscultatory D5 value minus direct DBP value) was variously reported as $+7.5 \mathrm{mmHg}(4),+12 \sim 20 \mathrm{mmHg}(5),-7 \mathrm{mmHg}$ $(8),+9 \mathrm{mmHg}(10)$, and $+13.1 \mathrm{mmHg}(11)$. In addition, the mean differences between auscultatory SBP and direct SBP were reported as $+4 \mathrm{mmHg}$ (2), $-2.6 \mathrm{mmHg}(3),-3 \mathrm{mmHg}(4),-2 \mathrm{mmHg}$ (5), $-12 \mathrm{mmHg}(8),-3 \sim 4 \mathrm{mmHg}(10)$, and $-24.6 \mathrm{mmHg}(11)$. Furthermore, auscultatory judgment of the DBP on the basis of cessation of Korotkoff sounds depends on experience and lacks a clear theoretical basis. The complicated relationship between optimum cuff width and the brachial circumference is another weak point of the auscultatory cuff method.

To solve these problems, we have developed a new noninvasive sphygmomanometer that employs hydraulic pressure instead of air pressure. Cuff pressure is measured at the center of the inner cuff

From the Second Department of Internal Medicine, Urafune Hospital of Yokohama City University, *Second Department of Internal Medicine, Yokohama City University, Yokohama, Japan.

Address for Reprints: Osamu Tochikubo, M.D., Second Department of Internal Medicine, Urafune Hospital of Yokohama City University, 3-46, Urafune-Cho, Minami-ku, Yokohama 232, Japan.

Received June 30, 1992; accepted in revised form October 26, 1992. 
which is placed on the skin above the artery. This report describes the new method and compares its accuracy with that of direct BP measurement as well as the auscultatory method.

\section{Subjects and Methods}

\section{Hydraulic-Cuff Method}

The novel feature of our hydraulic-cuff system is that it employs a liquid, and not air, to pressurize the cuff (Fig.1). When the BP is measured by using air, pressure oscillations occuring within the cuff due to the arterial pulse cannot be measured accurately up to their higher frequency components, because air has a high degree of compliance. In the case of a liquid, however, the effects of compliance can be ignored. Thus, to measure the arterial pressure changes as faithfully as possible, it is desirable to use a liquid-filled cuff.

The compliance of the rubber cuff generally employed in auscultatory equipment also poses a difficulty. Our cuff is of the usual width $(13 \mathrm{~cm})$, but it is made of a special low-compliance vinyl chloride mesh. The cuff is enclosed in a hard plastic cylindrical cover that permits movement in the direction of the circumference only. This is fixed in place by a soft adhesive cover that prevents outward expansion of cuff pressure. The entire cuff is formed as a single unit that is easy to wrap around the upper arm. Instead of measuring pressure within the outer cuff, which corresponds to the standard auscultatory cuff, the new device measures hydraulic pressure within an inner cuff $(3 \mathrm{~cm}$ wide and $6 \mathrm{~cm}$ long) situated inside of the center of the outer cuff and directly on the skin above the artery. The pressure acting on the artery is measured by a smll pressure sensor in the inner cuff. A tube $0.2 \mathrm{~mm}$ inside diameter and $10 \mathrm{~cm}$ long connects the outer and inner cuffs, and a special valve controls flow between the two.

A fixed-flow volume infusion pump (Nemoto Co., type B, Japan) is used to increase hydraulic pressure in this double cuff. Although the pump can vary the flow into the cuff from 0 to $99 \mathrm{ml} / \mathrm{min}$, it is adjusted to increase cuff pressure by about 3 $\mathrm{mmHg} / \mathrm{sec}$ and to infuse liquid at a rate of $50-80$ $\mathrm{ml} / \mathrm{min}$ at the time of measuring. The tube connecting the cuff and the pump is rigid and of low compliance. Inner-cuff pressure and its differential curve (dp/dt of the inner-cuff pressure) are recorded during pressurization. Recording is performed with an Omnicorder (NEC Sanei-Sha, SANEI-8M14, Japan).

\section{Measurement of Blood Pressure \\ Measuring $D B P$}

The infusion pump steadily elevates pressure within the double cuff. When the pressure exceeds about $10 \mathrm{mmHg}$, the inner-cuff pressure sensor detects arterial pulsations as oscillations. Below the DBP, although the absolute oscillation values are smaller, this produces a proportional curve that is almost identical with arterial pulsation, i.e., the descending segment (diastolic phase) and the ascending segment (systolic phase) form an angle like the nadir of an inverted triangle (complete oscillation). However, when inner-cuff pressure is higher than DBP, the descending segment of inner-cuff oscillations cannot descend all the way to the nadir (the descending segment of the curve is cut short). Consequently, when the inner-cuff pressure reaches or exceeds the DBP, a plateau forms between the descending and ascending segments of the oscillation curve (altered oscillation) (Figs.2, 3). Thus, the inner-cuff pressure between that of the last complete oscillation and the first altered oscillation is taken to represent the DBP.

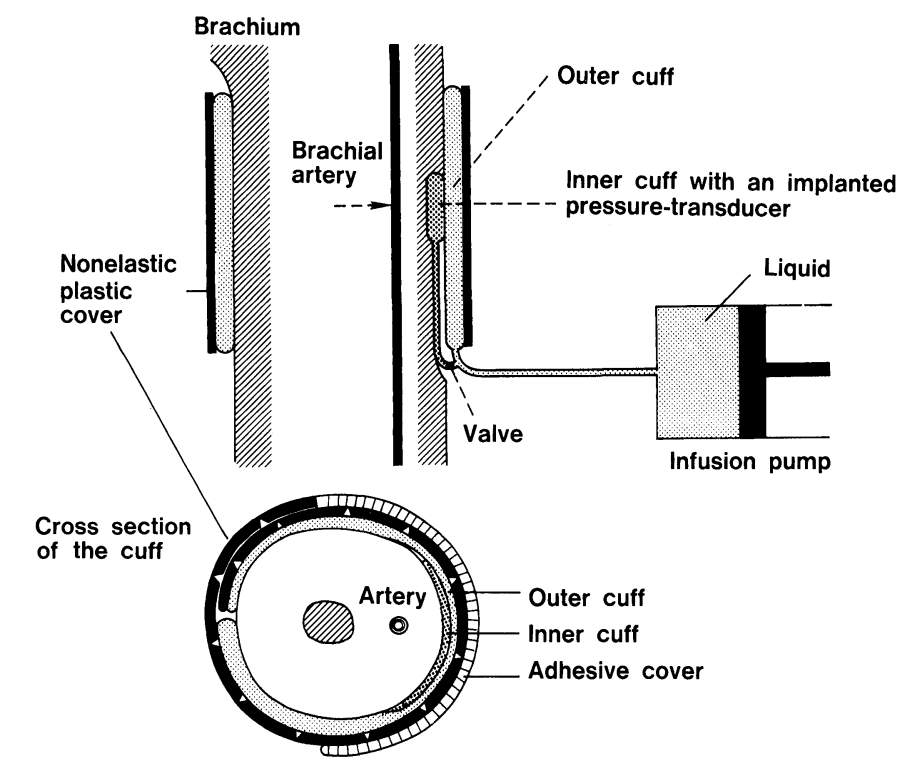

Fig. 1. Schematic diagram of the hydraulic-cuff method and the cross section of the double cuff for measuring blood pressure $(B P)$. The infusion pump applies hydraulic pressure to the double cuff (outer cuff and inner cuff with an inplanted pressure transducer) which is wrapped around the upper arm, and the change of the pulsatile patterns of the inner-cuff pressure is recorded to analyze $B P$. 


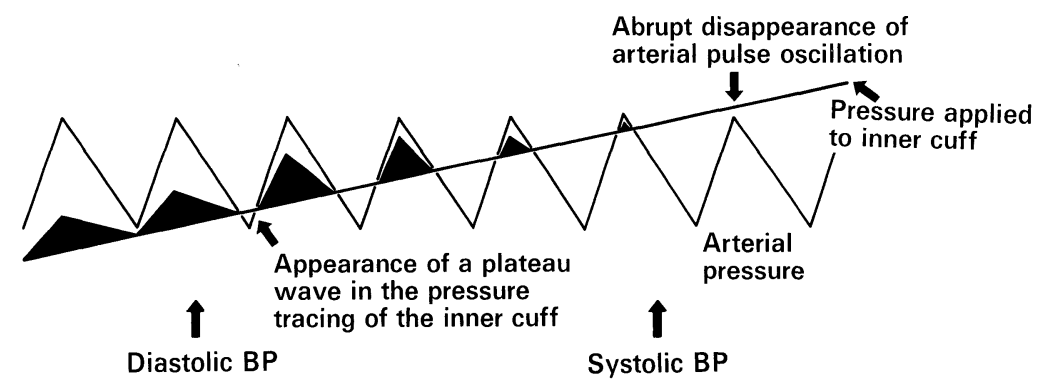

Fig. 2. The principles by which diastolic and systolic blood pressures (BP) are judged according to the hydraulic-cuff method. When inner-cuff pressure is higher than diastolic BP, a plateau appears at the end of the oscillation curve. Oscillations decrease suddenly above the systolic BP.

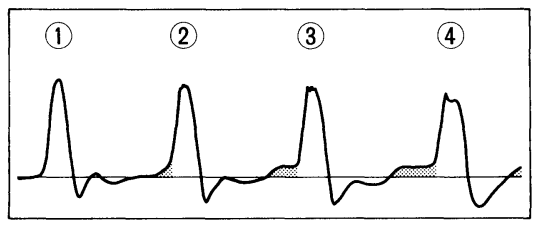

(1) (2) (3)

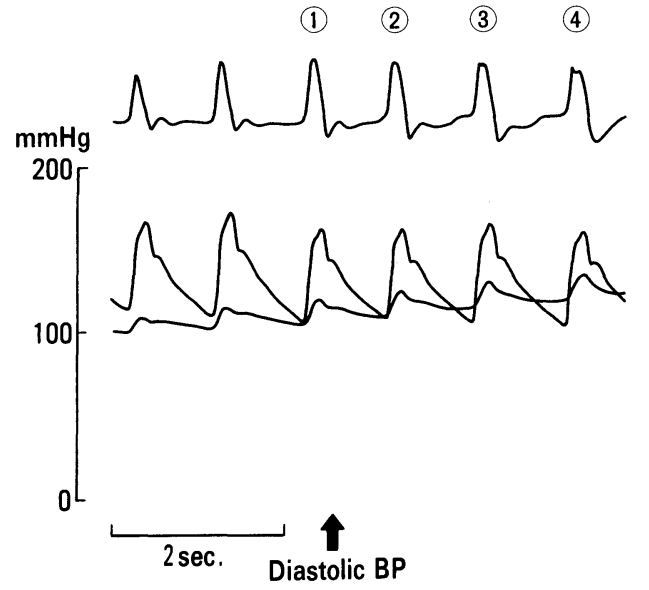

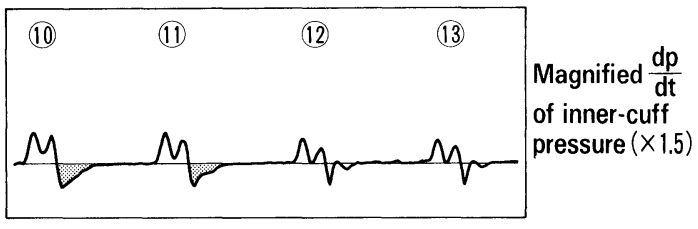
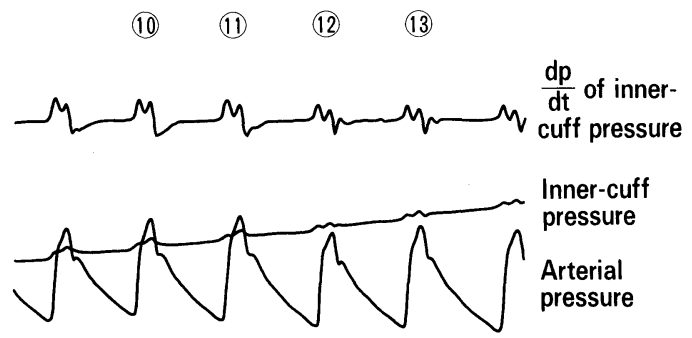

Systolic BP

Fig. 3. Reading blood pressure (BP) with the hydraulic-cuff system. The ascending segments of the inner-cuff pressure differential (dp/dt) are altered when cuff pressure exceeds the diastolic BP level (No. of differentials is 2, 3, 4). The oscillation pattern of the differentials changes suddenly above the systolic BP level (No. of differentials is 12, 13).

\section{Measuring $S B P$}

When cuff pressure is gradually increased to above the SBP level, it closes the artery directly below the inner cuff. This should cause a cessation of arterial pulse oscillations. However, since oscillation in the upstream position of the artery continues, the outer cuff still senses the arterial pulse, and this is transferred to the inner cuff causing slight oscillations. Since these are not being sensed directly by the inner cuff, the oscillation volume decreases suddenly. Both the inner and outer cuffs sense the arterial pulse simultaneously at levels below the SBP, so the inner-cuff pressure is a compound value derived from both cuffs. Thus, the point where the arterial pulsation curve decreases dramatically and suddenly alters form is taken to represent the SBP (Figs. 2, 3). Omnicorder recordings and a microcomputer (NEC PC9801) were used together to analyze these curves (Fig. 4) and the process is completed in less than a minute.

\section{Evaluation of the Accuracy of the Hydraulic-Cuff Method}

We studied 7 normotensive volunteers and 14 hospitalized patients with essential hypertension. The normotensive subjects (6 males and 1 female) had a mean age of $36 \pm 17$ (SD) years (4 males were under 25 years old), a mean height of $169 \pm 7.8 \mathrm{~cm}$, a mean weight of $65.4 \pm 5.9 \mathrm{~kg}$, and a mean upper arm circumference of $26 \pm 3.0 \mathrm{~cm}$. The hypertensive patients ( 7 males and 7 females) had a mean age of $46 \pm 13$ years, a mean height of $160 \pm 7.0$ $\mathrm{cm}$, a mean weight of $65.8 \pm 12.8 \mathrm{~kg}$, and a mean upper arm circumference of $28.0 \pm 2.6 \mathrm{~cm}$ (the arm circumferences of 3 hypertensives were more than $30 \mathrm{~cm}$ ). Measurements were performed after in- 


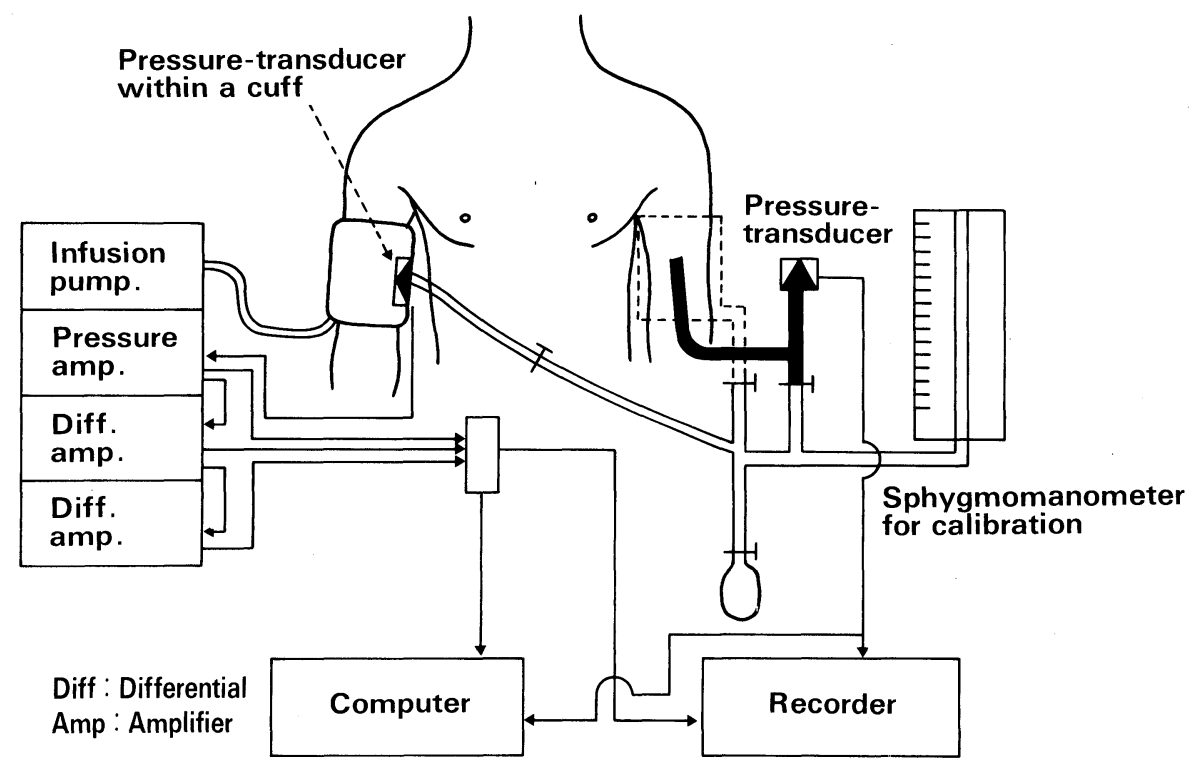

Fig. 4. Recording system for the comparison between direct blood pressure values (right) and blood pressures obtained by the hydraulic-cuff or auscultatory methods (left).

formed consent to the study protocol was obtained.

For direct measurements, subjects were requested to lie in the supine position. According to the Seldinger technique, a 20-gauge arterial catheter was inserted into the left brachial artery and was connected to a $15 \mathrm{~cm}$ long noncomplaint extension tube. Blood pressure was measured directly using a Gould P50 transducer (Spectramed, CA) fixed to the upper arm. The resonance frequency of the recording system was about $30 \mathrm{~Hz}$. In order to compare the hydraulic-cuff and direct methods, a hydraulic cuff was wrapped around the right upper arm of each subject. DBP and SBP were measured 4 times each. Inner-cuff pressure was initially raised to $40 \mathrm{mmHg}$ within $2-3 \mathrm{~s}$. Thereafter, pressurization continued at a rate of from $2-4 \mathrm{mmHg} / \mathrm{s}$. After the oscillations suddenly decreased and altered their form, $20 \mathrm{mmHg}$ of additional pressure was applied.

The direct arterial pressure, the inner-cuff pressure, and the differential $(\mathrm{dp} / \mathrm{dt})$ of the pressure oscillation were recorded (Fig. 4). In the case of direct measurement, the SBP value was obtained by averaging the 2 peak values of the arterial pressure curves on either side of the point at which the indirect SBP judgment was made. Likewise, direct DBP was determined by taking the mean value of the 2 curve nadirs on either side of the indirect DBP point.

After these measurements had been completed, a conventional auscultatory cuff $(13 \mathrm{~cm}$ wide) was wrapped around the right upper arm of each subject. During auscultatory measurements, arterial pressure and cuff pressure were simultaneously recorded. Measurements were taken twice each by 2 doctors in each study.

Statistical analysis of all data was performed using Student's paired $t$-test (two-tailed) and a nonparametric unpaired $t$-test to compare the 2 groups. In addition, averages were obtained for the differences between 2 methods and individual variables were compared using linear regression analysis. Data are presented as the mean and standard deviation (SD).

\section{Results}

Accuracy of the Hydraulic-Cuff Method

SBP measured by the hydraulic-cuff method was designated as SBPi and the SBP by the direct method was referred to as SBPd. The difference between them was defined as $E(E=\mathrm{SBPi}-\mathrm{SBPd})$. In 84 measurements, the correlation between SBPi and SBPd was very strong $(r=0.99)$ and the mean difference $(E)$ was $-0.74 \pm 2.3 \mathrm{mmHg}$ (Fig. 5). In the case of DBP (DBPi and DBPd), the correlation was also strong $(r=0.98)$ and the mean difference $(E=$ DBPi-DBPd) was $0.94 \pm 3.2 \mathrm{mmHg}$ (Fig. 5).

\section{Comparison with the Auscultatory Method}

The hydraulic-cuff method and the auscultatory method were used to obtain 4 measurements each. The coefficient of variation for $E$ values of the same subjects was $58 \pm 15 \% / 34 \pm 14 \%$ (SBP/DBP) for the hydraulic cuff method and $33 \pm 11 \%$ / $51 \pm$ $25 \%$ for the auscultatory method. Averages were taken and compared with values obtained with the direct method The mean difference of SBP values measured by the auscultatory and direct methods was $-6.8 \pm 4.0 \mathrm{mmHg}$. The auscultatory method gave significantly lower values than the direct method $(p<0.01)$. In the case of DBP, the mean difference between the 2 methods was $1.1 \pm 6.5$ $\mathrm{mmHg}$. In the 3 obese subjects with upper arms $>$ $30 \mathrm{~cm}$ in circumference, auscultatory measurements tended to be higher. They tended to be low, however, in the 4 subjects under 25 years of age (Fig. 6).

The differences between values obtained by the 
Systolic BP

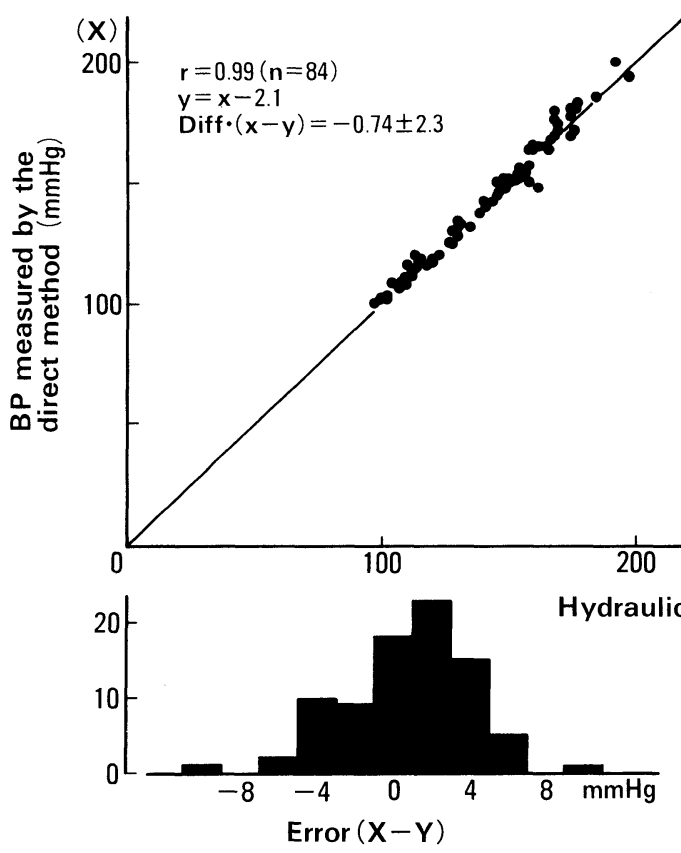

Diastolic BP

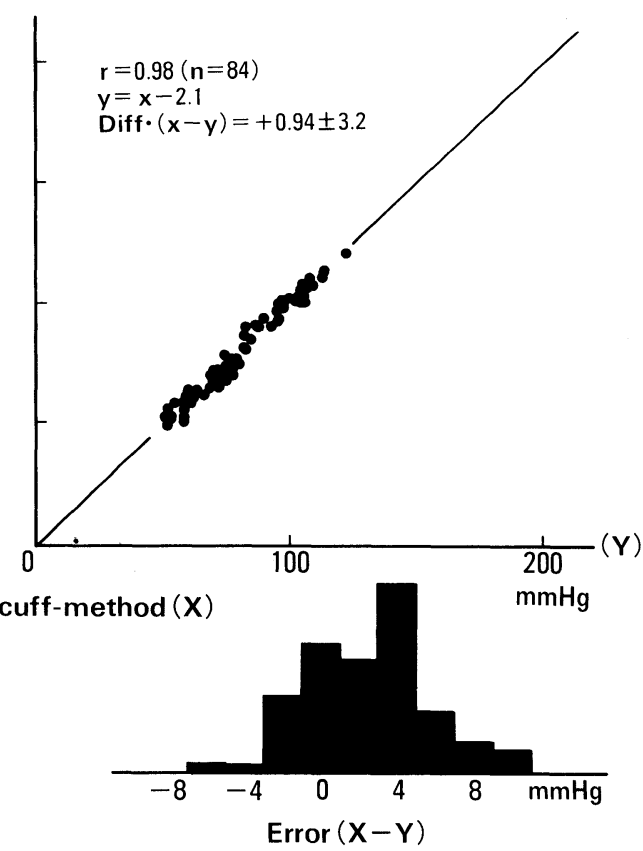

Fig. 5. Comparison between blood pressure (BP) values obtained with the direct and hydraulic cuff methods. Errors $(X-Y)$ were derived from the difference between the hydraulic cuff method $(X)$ and the direct method $(Y)$.
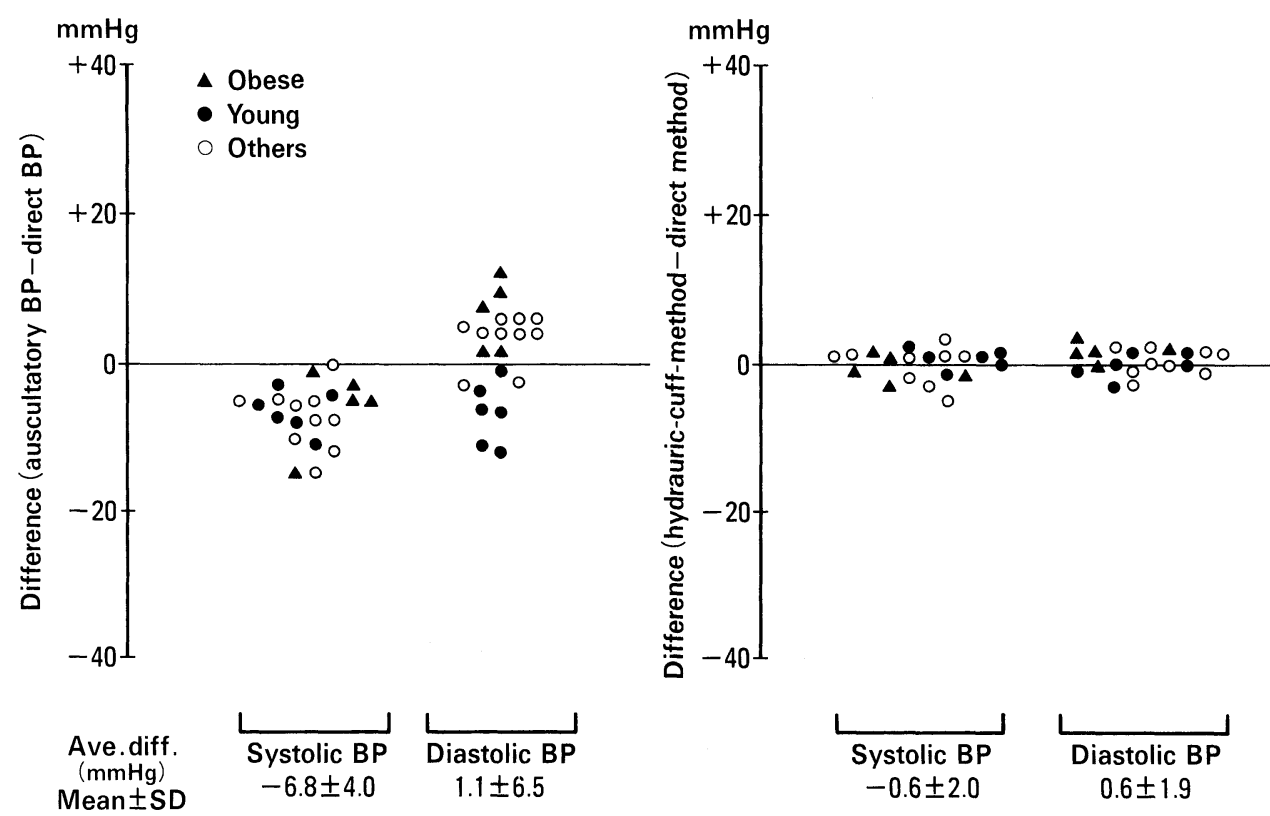

Fig. 6. Accuracy of the auscultatory and hydraulic-cuff methods. Measurements were taken twice each by 2 doctors and the averages of the 4 values were obtained (the left panel: the differences in BP between the auscultatory and direct methods, the right panel: the differences in BP between the hydraulic cuff and direct methods).

hydraulic-cuff and direct methods (mean $E$ of SBP; $-0.6 \pm 2 \mathrm{mmHg}$, mean $E$ of DBP; $0.6 \pm 2 \mathrm{mmHg}$ ) were significantly smaller than those between measurements obtained by the auscultatory and direct methods $(p<0.05)$ (Fig. 6).

\section{Discussion}

There are discrepancies between direct and indirect blood pressure measurements. The auscultatory BP is estimated on the basis of Korotkoff sounds, which are generated from turbulent blood flow distal to 
the compressed artery and transmitted through the tissues. The auscultatory method tends to give low SBP values (2-6). In addition, when determination is made at D5 under conditions of rapid blood flow, it tends to give low DBP values as well. Indeed, it is not unusual to have an error of more than \pm 8 $\operatorname{mmHg}(4,5,10,11)$.

Of course, the auscultatory method is simple and inexpensive, and slight measurement errors may be of no clinical importance. However, the mangement of mild hypertension has increased recently, making more accurate BP measurement necessary. There are 10-20 million patients with mild hypertension in Japan and the DBP of mild hypertensives ranges no more than $15 \mathrm{mmHg}$ (DBP values range from 90 to $104 \mathrm{mmHg}$ ). Consequently, an error of $1 \mathrm{mmHg}$ could influence the diagnosis of hypertension for $a b-$ out one million people. This is an extreme example, but as long as the diagnosis of hypertension depends on BP values, the maximum possible accuracy of measurement is desirable. Hopefully, measurements should be within an error range of \pm 4 $\mathrm{mmHg}$. However, the auscultatory method often produces larger errors.

Another serious weakness of the auscultatory method involves using Korotkoff sounds. In spite of the vast amount of research $(1,12-14)$ devoted to the topic, no clear conclusion has yet been drawn as to why this practice is theoretically followed. There is a long-standing dispute over whether to use the point at which the sounds are muffled (D4) or the point at which they cease entirely (D5) as an indication of DBP. In some countries, the muffling point is still the basis of judgment, but the American Heart Association (9) indicates D5 as the best estimate of indirect DBP. In the case of young hypertensive subjects, whose cardiac output is comparatively high and whose blood flow is rapid, auscultatory measurement of DBP is clearly lower than the direct DBP. Similar phenomena are noted in infants, pregnant women, and patients with hyperthyroidism or anemia. In such instances, we are advised to record D4 and D5 simultaneously, but the diseases and symptoms for which it is better to judge DBP on the basis of D4 can not be determined practically.

In addition, the hearing power of the individual performing the examination greatly influences the determination of D5. Three basic factors compose Korotkoff sounds; volume, frequency, and timbre. The intensity of volume is continuous and the presence or absence of sound cannot be simply determined. This causes difficulties in automating the auscultatory method. For instance, if the sensitivity of the microphone is enhanced, Korotkoff sounds can be detected much lower than the direct DBP. Consequently, objectively determining the microphone voltage level at which Korotkoff sounds cease becomes difficult. We are forced to conclude that, because of its inherent problems, the auscultatory method of measuring blood pressure is difficult to describe as logical.

Many of the automated measuring devices that have recently become popular use air pressure and the oscillation method to determine the BP. However, because air is employed to fill the cuff, the oscillation differs from the arterial pulse curve. As is true of the auscultatory method, the oscillation method produces large measurement errors, and the theoretical grounds used for determination of the DBP are vague (20).

With our hydraulic-cuff method, the cuff itself has been improved. BP is measured by an inner cuff and the BP is read while the cuff pressure is rising. In contrast, cuff pressure must be raised much higher than the SBP, which is read as cuff pressure drops with the auscultatory method. Because it is impossible to know how high to raise the pressure beforehand, excess pressurization is necessary. This is not the case with the hydraulic-cuff method, which also takes less time than the auscultatory method because both DBP and SBP are read during the pressurization process.

Moreover, unlike the auscultatory and oscillometric methods, the hydraulic cuff method has a firm theoretical basis and produces less error. However, the measurement process is complicated since the inner-cuff pressure must be fed into a recorder to make it possible to read blood pressure from the pressure curve patterns. To solve this problem, we are currently developing a computer program that will make automatic judgments possible. This should allow the hydraulic-cuff method to become as simple and rapid as the already existing automatic devices.

In concluding, we should like to remark that the hydraulic-cuff method for measuring blood pressure is theoretically clear. In addition, it is clinically valuable because it is more accurate than the auscultatory method. However, investigation of more subjects with narrow and obese arms is necessary to validate our method.

\section{References}

1. Geddes LA: The direct and indirect measurement of blood pressure. Year Book Medical Publishers, Chicago, 1970.

2. Forsberg SA, De Guzman M, Berlind S: Validity of blood pressure measurement with cuff in the arm and forearm. Acta Med Scand 1970; 188: 389-396.

3. Goldstein S, Killip T: Comparison of direct and indirect arterial pressures in aortic regurgitation. $N$ Engl J Med 1962; 267:1121-1124.

4. Karvonen MJ, Telivuo LJ, Järvinen EJK: Sphygmomanometer cuff size and the accuracy of indirect measurement of blood pressure. Am J Cardiol 1964; 13: $688-693$.

5. London SB, London RE: Comparison of indirect pressure measurements (Korotkoff) with simultaneous direct brachial artery pressure distal to the cuff. Adv Intern Med 1967; 13: 127-142.

6. Nielsen PE, Janniche $H$ : The accuracy of auscultatory measurement of arm blood pressure in very obese subjects. Acta Med Scand 1974; 195: 403-409.

7. Ragan C, Bordley J: The accuracy of clinical measurements of arterial blood pressure with a note on the auscultatory gap. Bull John Hopkins Hosp 1941; 69: 504-528.

8. Roberts LN, Smiley JR, Manning GW: A compari- 
son of direct and indirect blood pressure determinations. Circulation 1953; 8: 232-242.

9. Frohlich ED, Grim C, Labarthe DR, Maxwell MH, Perloff D, Weidman WH: Recommendations for human blood pressure determination by sphygmo-manometers. Report of a special task force appointed by the steering committee, American Heart Association. Hypertension 1988; 11: 210A-222A.

10. Hamilton WF, Woodbury RA, Harper HT: Physiologic relationships between intrathoracic, intraspinal, and arterial pressures. JAMA 1936; 107: 853-856.

11. Holland WW, Humerfelt S: Measurement of blood pressure: comparison of intra-arterial and cuff values. Br Med J 1964; 2: 1241-1243.

12. Gitting JC: Auscultatory blood-pressure determination. Arch Intern Med 1910; 6: 196-204.

13. Chungcharoen D: Genesis of the Korotkoff sounds. Am J Physiol 1964; 207: 190-194.

14. McCutcheon EP, Rushmer RF: Korotkoff sounds. An experimental critique. Circ Res 1967; 20: 149-161.
15. WHO: Report of a WHO Expert Committee: Arterial hypertension. WHO Tech Rep Ser 1978; 628: 7.

16. WHO/ISH: The 1986 guidelines for the treatment of mild hypertension: memorandum from a WHO/ISH meeting. WHO Bulletin, Geneva 1986; 31-35.

17. Rebenson-Piano M, Holm K, Powers M: An examination of the differences that occur between direct and indirect blood pressure measurement. Heart \& Lung 1987; 16: 285-294.

18. Rebenson-Piano M, Holm K, Foreman MD, Kirchhoff KT: An evaluation of two indirect methods of blood pressure measurement in ill patients. Nurs Res 1988; 38: 42-45.

19. Gravlee GP, Brockschmidt JK: Accuracy of four indirect methods of blood pressure measurement, with hemodynamic correlations. J Clin Monitoring 1990; 6: $284-298$.

20. Mauck GB, Smith CR, Geddes LR, Bourland JD: The meaning of the point of maximum oscillations in cuff pressure in the indirect measuring blood pressure. II. J Biomech Eng 1980; 102: 28-33. 